\title{
Análisis de las expectativas del profesorado novel sobre su futura labor docente
}

\author{
Ma del Valle Cecilia Montilla Coronado, Pablo Maraver-López, Cristina Romero Oliva y Ariadna Martín \\ Montilla
}

Universidad de Huelva

\section{RESUMEN}

Dado el carácter cambiante de las leyes educativas y los cambios prescriptivos en el acceso a la profesión docente, esta investigación se plantea conocer y describir las distintas concepciones del profesorado novel que cursó el Máster de Profesorado, durante tres cursos académicos consecutivos en la Universidad de Huelva. Los objetivos planteados tratan de desvelar los planteamientos didácticos de 323 titulados universitarios, así como sus expectativas profesionales sobre la docencia. Se trata de conocer cómo piensan plantear sus objetivos docentes, las actividades de clase, la evaluación así como los recursos que consideran utilizar en su quehacer profesional. La metodología es transversal. Una de las conclusiones más interesantes es que una mayoría del profesorado novel se plantea metodologías innovadoras y participativas, encuadrándose en un paradigma constructivista que permita, a través de los variados conocimientos previos del alumnado, progresar en función de sus particularidades. Asimismo la utilidad y uso que se reconocen hacia la evaluación es continua y entendida como un mecanismo que permita progresar más que como calificación final. Las ventajas más destacadas de esta profesión docente la ven en la satisfacción personal y la gratificación que proporciona enseñar. Por otro lado, el inconveniente más destacado es la falta de respeto del alumnado.

Palabras Clave: expectativas docentes, profesorado novel, metodología docente, estrategias curriculares.

\section{Pre-service teachers' expectations of their future labor}

\section{ABSTRACT}

Given the changing pattern of educational laws and prescriptive changes in the access to the teaching profession, this research aims to know and describe the different conceptions of pre-service teachers, who attended the Master's Degree, during three consecutive academic courses at the University of Huelva. The objectives set out to reveal the didactic approaches of 323 university graduates, as well as their professional expectations on teaching. It is about knowing how they plan to set their teaching objectives, class activities, evaluation as well as the resources they consider to use in their professional work. The methodology is transversal. One of the most interesting conclusions is that a majority of new teachers consider innovative and participatory methodologies, framed in a constructivist paradigm that allows, through various prior knowledge of students, to progress according to their particularities. Likewise, the usefulness and use that are recognized towards the evaluation is continuous and understood as a mechanism that allows progress rather than as a final qualification. The most outstanding advantages of this teaching profession are seen in the personal satisfaction and the gratification that teaching provides. On the other hand, the most notable drawback is the lack of respect from the students.

Keywords: teachers' expectations, pre-service teachers, teaching methodology, curricular strategies.

\section{Introducción}

\section{Distintos paradigmas de estudio}

Una de las líneas actuales de investigación dentro del campo de la docencia, se refiere a las concepciones de este colectivo acerca de su trabajo desde el punto de vista de los enfoques basados en el propio profesorado. En él se parte de la propia concepción y expectativas de desarrollo profesional; y se pretende analizar la estructura latente que explica y da sentido a la enseñanza para desde ella, explicar el comportamiento del docente (Doménech y Gómez, 2003).

El paradigma mediacional centrado en el profesor supuso la superación de los paradigmas anteriores; ante la imposibilidad de llegar a conclusiones contundentes por el hecho de que no contemplaba la flexibilidad del proceso. En este paradigma, el centro de atención lo constituye el ámbito de la enseñanza, entendida como un proceso de planificación y toma de decisiones 
del docente tal que profesional cualificado. Aquí, el objeto de estudio son los procesos complejos de pensamiento. En este sentido, "el comportamiento es, en gran medida, el resultado del pensamiento del profesor: de sus conocimientos, de sus estrategias para procesar la información y utilizarla en la resolución de problemas y de sus actitudes y disposiciones personales" (Pérez Gómez, 1987, p. 115).

El trabajo que aquí se presenta se centra en este paradigma y no contempla variables relacionadas con el complementario, surgido en la misma época, el paradigma mediacional centrado en el alumnado. El paradigma ecológico incorpora la consideración de las variables contextuales que enmarcan el trabajo docente pero que sería objeto de estudio posterior, como evolución de estos planteamientos iniciales. Dentro de este paradigma existen tres dimensiones de la investigación educativa: la planificación docente, las decisiones y pensamientos interactivos y sus teorías y creencias. A estas tres corrientes de investigación clásicas se pueden añadir otras dos en las que se ha profundizado más recientemente: las diferencias entre el profesorado experto y novel. Es en la primera categoría donde enmarcamos nuestro estudio y en el sector de profesorado novel.

La planificación docente como garantía de eficacia educativa, se ha entendido desde dos perspectivas distintas que han dado lugar a la utilización de metodologías diferentes. Por un lado la perspectiva cognitiva, desde la cual la planificación supone la activación de una serie de procesos psicológicos mediante los que la persona en cuestión participa directa y personalmente en la enseñanza, analizando los medios y fines que le permitan crear el marco de actividad futura, funcional y por otro lado la perspectiva conductista, inserta en modelos transmisivos. Dicho de otro modo, docente encontramos básicamente dos grupos de docentes: aquellos que muestran creencias tradicionales y aquellos que se reconocen progresistas. Los docentes considerados tradicionales suelen presentar creencias controladoras y organizan los contenidos de tal forma que el alumnado no requiera interpretarlos, o sea de modo cerrado, mientras que el profesorado progresista presenta creencias relativas y buscan la implicación, interpretación y estructuración del trabajo por parte del alumnado de cara a un aprendizaje significativo. Suelen crear situaciones de aprendizaje abiertas y "por hacer", dejando este espacio al alumnado.

Este paradigma más actual es fundamental para conocer y caracterizar las decisiones que un profesor o profesora toma para organizar su docencia. Existen pocas investigaciones al respecto dada la dificultad del conocimiento explícito de creencias, expectativas, teorías etc., que es el marco de referencia del profesorado pero que no se expresa de una forma precisa. En la muestra de estudiantes del Certificado de Aptitud Pedagógica (CAP), ahora Máster en Secundaria, estudiada por Doménech y Gómez, (2003), la mayoría del profesorado se enmarcaba en el paradigma cognitivo y humanista, marcando una tendencia progresista e idealista que, luego en la práctica, puede girar en otro sentido (Genovard y Gotzens, 1990).

\section{Aspectos docentes que condicionan su trabajo}

Según Melief, Tigchelaar y Korthagen (2010), es necesario establecer una relación directa entre tres aspectos que condicionan las acciones de un docente: los conocimientos teóricos adquiridos durante su formación inicial y continua, el adquirido en la práctica y los condicionantes particulares: teorías implícitas, conceptos subjetivos, experiencias personales o personalidad.

En el estudio de Clara (2016), la mayor parte de los docentes prefieren enseñar guiados, en relativa consonancia, por sus preferencias de aprendizaje. Por su parte, Shulman (1990) establece que los docentes disponen de tres tipos de conocimiento: conocimiento proposicional, conocimiento de casos y conocimiento estratégico. El segundo corresponde al conocimiento práctico y comprende:

1) El conocimiento sobre el contenido a enseñar (los hechos y conceptos más significativos de un cada área) y se refiere igualmente a otros ámbitos como la forma en que se planifica y se lleva a cabo la enseñanza, o sea, la metodología.

2) El conocimiento pedagógico general (creencias y opiniones sobre la enseñanza y el aprendizaje, condiciones del alumnado...).

3) El conocimiento sobre el currículum.

4) El conocimiento pedagógico: cómo adaptar y hacer apropiada la materia al alumnado y al contexto. Está compuesto, por tanto, de representaciones de las estrategias instruccionales más útiles para hacer asequible el contenido a enseñar.

5) El conocimiento sobre el alumnado.

6) El conocimiento del contexto educativo.

7) El conocimiento de los fines, propósitos y valores educativos.

Con respecto a los estudios que comparan profesores noveles y con experiencia, fundamentalmente se centran en diversos aspectos como la planificación, creencias, principios didácticos, la toma de decisiones, su conducta durante la práctica interactiva, etc., pero son escasos los estudios que analicen la evolución y proceso de cambio de docentes desde su condición de principiantes a expertos (González, 2012); hay que decir que en la mayoría de estudios y en el nuestro propiamente, se identifica el profesorado novel con el que tiene ninguna o poca experiencia que es justamente la población que nos encontramos en el Máster prescriptivo para el comienzo de la docencia. Entendiendo la formación inicial como un conjunto de acontecimientos, conocimiento y vivencias que predisponen al futuro profesorado a adquirir actitudes, normas, posicionamientos profesionales necesarios para un adecuado desempeño profesional, pretendemos relacionar dichas características de partida con planteamientos docentes futuros, clima de centro, expectativas acerca del funcionamiento del mismo, actitudes del alumnado, etc.

También se han publicado estudios recientes que muestran la opinión del alumnado del Grado en Educación sobre las competencias que configuran el perfil del buen docente como el de Valdemoros-San Emeterio y Lucas-Molina (2014). Por su parte, la revisión bibliográfica más importante y extensa dentro de la línea de investigación de preocupaciones del profesorado novel es la realizada por Veenman $(1984,1988)$. Este autor recogió 91 estudios internacionales realizados desde 1960 sobre la problemática del profesorado principiante y encontró grandes coincidencias en dichas preocupaciones: la disciplina y la motivación, en primer y segundo lugar. En el sexto y séptimo puesto los materiales y la organización del trabajo. Distintas son las clasificaciones de problemáticas más acuciantes en el profesorado encontradas por Marcelo (1993), Mayor Ruiz (1996) o González-Granda, Álvarez-Menéndez y González-Pienda (1991). Estos últimos autores encuentran que existe un cambio importante de actitud en el profesorado novel cuando comienza su práctica, fundamentalmente debido a que se olvidan las creencias y posicionamientos por la necesidad de resolver las realidades presentadas cada día en el aula.

Este estudio pretende arrojar originalidad en el tratamiento de aspectos relacionados con la metodología docente y didáctica 
de las distintas materias curriculares describiendo los posicionamientos del profesorado futuro. Respecto a este aspecto, se observa escasez de estudios sobre los puntos de partida respecto a las distintas metodologías docentes, las estrategias para el tratamiento de los elementos de la programación, en los programas de formación para profesorado novel, por ejemplo el llevado a cabo por Negrillo e Iranzo (2009), titulado "Comencem bé". En él se recogen aspectos como: trabajo en equipo, organización y funcionamiento del centro, desarrollo personal y profesional, desarrollo de materiales curriculares, TICs, Tutoría, atención a la diversidad e interculturalidad, gestión del aula, etc. Las expectativas de los participantes era conseguir una adecuada competencia docente que permita llevar a cabo un proceso de enseñanza-aprendizaje innovador teniendo en cuenta el desarrollo curricular y que promueva el aprendizaje autónomo en el alumnado. Así como aprender a utilizar una metodología activa en contextos y grupos diversos.

Utilizando los ciclos de experiencia de Kelly (1955), y tomando como temas principales el desempeño de la correcta planificación y organización de las clases, la capacidad de responder adecuadamente a las dudas del alumnado y la formación del proceso de evaluación del mismo, García, Orellana y López-Cepero (2010) concluyen que en el profesorado novel resalta como cualidad principal y más importante la coherencia personal, de forma que se supla las posibles carencias del profesorado que apenas ha acumulado experiencia con una imagen afín y coherente a las expectativas creadas, siendo capaz de transmitirlas al alumnado, convirtiéndose de este modo, no solo en modelo profesional sino también personal que se permite errar e instruir en un adecuado modelo de enseñanza-aprendizaje que se ajuste a sus necesidades, huyendo de esta manera de la imagen del perfecto profesor magistral que todo lo sabe.

Por su parte, Zeichner $(1983,1993)$ apuesta porque el profesorado novel no se limite a la observación de la enseñanza sino que crea que debe implicarse en la comprensión del centro educativo como organización y en la influencia que ejerce. Desde esta comprensión, los noveles podrán dar coherencia y sentido a su tarea, su responsabilidad, la problemática y limitaciones con que se enfrenta, así como posibilitar una acción reflexiva y un cambio importante y veraz en el proceso educativo.

Nos posicionamos junto a Azcárate y Cuesta (2005) en que la etapa inicial de trabajo docente, sin reflexión sobre una práctica pedagógica específica, sin poseer una formación pedagógica sólida, pero permeables y susceptibles de moldeamiento, es crucial para determinar una mirada amplia y sin "contaminación" sobre la tarea docente en aras de plantear una enseñanza verdaderamente sustentada en ilusión y nuevos planteamientos innovadores que no renuncien a las características teóricas estudiadas.

Ya desde muy atrás se planteaban las principales preocupaciones del profesorado novel. Fuller y Brown (1975) plantearon cuatro niveles de preocupaciones: identificación y acercamiento al alumnado para poder gestionar la disciplina, exploración de estrategias profesionales y organización efectiva de la docencia, las relaciones con las familias y, por último, las relaciones con el centro y su funcionamiento. Asimismo, y según Whitworth (1996) sentirse a gusto con la materia y la preocupación por la dirección del aula y el control del alumnado son las máximas inquietudes en el profesorado novel.

Por otro lado, Marcelo (1987) consideró que las creencias del profesorado, las características del alumnado y el libro de texto utilizado influyen en la planificación de una forma totalmente lineal al igual que Capell (1998). Al-Musawi (2001) estudió los dilemas sobre la enseñanza y el aprendizaje del profesorado novel, concretamente el conocimiento sobre el currículum, el rol del profesorado y la relación alumnado-profesorado, concluyendo que las creencias de participantes en el programa de prácticas, no cambiaron de manera considerable adquiriendo, obviamente, una percepción más realista del trabajo de enseñar.

Todos los planteamientos realizados hasta el momento dan cuenta de la importancia de conocer la situación inicial del profesorado sin experiencia para poder describir, en un primer momento y posteriormente mejorar, las estrategias metodológicas planteadas, la estructura de tareas que piensan llevar a cabo en sus aulas, los criterios que le llevan a seleccionar y organizar ciertos tipos de actividades, así como los recursos necesarios para ello. Es por ello que, consideramos relevante este conocimiento inicial del que poco se sabe, antes de consolidar prácticas desfasadas, poco apropiadas o inadecuadas a la realidad del alumnado. El docente experimentará un alto grado de coherencia y satisfacción profesional cuando consiga llevar al mismo grado la formación y éxitos docentes y su propia auto-realización profesional.

En un estudio realizado sobre creencias del alumnado que se encuentra iniciando los cursos de formación inicial del profesorado (Sánchez y Boix, 2008) se les preguntó qué competencias creían que resultaban más adecuadas para ser un/a buen/a docente, siendo la respuesta más común la transmisión de valores como el esfuerzo y la comunicación como una estrategia de aproximación al currículum, así como la adecuación a las necesidades educativas de todo el alumnado y al cambio desde la innovación educativa. En definitiva las competencias personales relacionadas con el "saber ser", como la coherencia, la paciencia, la empatía, la confianza, la autoridad reconocida, la capacidad de facilitar y mediar, la vocación y la facilidad de ser asequible en el trato con su alumnado.

La enseñanza, como consideración general, suele constituirse con la observación de los miembros de la profesión cuando trabajan. Este aprendizaje observacional se mediatiza por la predisposición personal, la idea sobre la enseñanza, el aprendizaje y la significación del rol docente (Marcelo, 2008). Esto explicaría las rutinas recurrentes de la gran mayoría de docentes en la planificación y desarrollo de sus clases. Este constructo personal y las concepciones educativas influyen en las innovaciones curriculares, además de haber diferencias en cuanto a la frecuencia, antecedentes y contenidos de las decisiones interactivas, respecto de profesorado novel y experto (Casado, 2007).

Así pues, parece que la enseñanza es una profesión en la que los aspirantes entran con creencias y concepciones preconcebidas sobre ella adquiridas a lo largo de sus años como estudiantes en el Sistema Educativo.

Otro aspecto importante que inquieta al profesorado novel es la situación que se va a encontrar en los centros a los que vaya. Sus características en cuanto a la ubicación, tamaño, peculiaridades educativas, composición del claustro, relaciones personales y cultura organizativa modelan también las expectativas, creencias y planteamientos docentes y personales; aspectos todos para los que nunca se ha preparado (Azcárate y Cuesta, 2005).

Acerca de la importancia sobre cómo el profesorado novel se plantea sus comienzos docentes, se encuentra un estudio llevado a cabo con 187 profesores y profesoras que cursaban el CAP. En esa ocasión se les preguntó sobre cuáles son sus valores y se concluye que la actitud personal del profesorado marca su posterior acercamiento a los centros así como las relaciones con el alumnado y demás miembros de la Comunidad Educativa (Cámara, 2010).

Una de las conclusiones obtenidas por Sánchez y Boix (2008) fue que el profesorado en formación para la futura docencia opina que las competencias más adecuadas para ser un buen docen- 
te están relacionadas con las competencias metodológicas, tales como la diversificación psicopedagógica, la adecuada transmisión del mensaje para su adecuada comprensión, despertar los intereses de los aprendices favoreciendo una clase activa, aplicación de una pedagogía activa y reflexiva, planificación de los aprendizajes y diseño de las clases utilizando las tecnologías de la información y comunicación (TICs) y las de autoaprendizaje y trabajo cooperativo. El profesorado novel en esa investigación consideraba que su participación en el centro educativo quedaría exclusivamente reducida a su acción docente dentro del aula, por lo que se supone que no tienen la visión global del centro como una sociedad de participación educativa.

Lo cierto es que las primeras experiencias en la profesión docente marcan en gran medida el desempeño futuro, y no siempre para bien. Resultan evidentes sus consecuencias para la calidad de la enseñanza, el clima de los centros, la moral del profesorado repercutiendo de forma importante en los resultados del alumnado, con el riesgo añadido de perder el potencial renovador que supone para el sistema educativo la incorporación de docentes jóvenes, ilusionados y motivados (Beca y Boerr, 2008).

Ante esta realidad, tal y como comentamos anteriormente, los futuros profesores deben contar con preparación suficiente para afrontar los retos que la sociedad actual impone. Para ello, además del compromiso personal y la voluntad por permanecer en un proceso constante de aprendizaje y renovación, las administraciones educativas deben hacerse eco de esta necesidad formativa del profesorado dotando de los recursos necesarios, así como proveyendo de planes de estudios adecuados, enfocados a la práctica y orientados a los destinatarios finales de la formación, el propio alumnado. Sólo de este modo lograremos aportar una formación integral y coherente con el mundo en que el individuo de desarrolla y participa activamente.

El objetivo principal de esta investigación es conocer las intenciones metodológicas en el profesorado novel, sus expectativas y concepciones sobre los centros y sus futuras prácticas docentes. Para ello se plantean los siguientes objetivos específicos:

1. Analizar los distintos elementos didácticos que plantea el profesorado novel: objetivos, actividades, contenidos y recursos didácticos.

2. Caracterizar las expectativas del profesorado novel sobre evaluación educativa.

3. Identificar las ventajas e inconvenientes de la profesión docente según el profesorado novel.

\section{Método}

\section{Procedimiento}

Esta experiencia se ha llevado a cabo en un contexto universitario de posgrado. Para el procedimiento de recogida de datos se repartieron los cuestionarios a todo el alumnado matriculado en el Máster Universitario en Profesorado de Educación Secundaria Obligatoria y Bachillerato, Formación Profesional y Enseñanza de Idiomas de la Universidad de Huelva durante 3 cursos académicos consecutivos, cursos 2012-13, 2013-14 y 2014-15. Una vez recogidos los datos fueron analizados en SPSS v.21.

\section{Instrumentos}

Para esta investigación se elaboró un Cuestionario ad hoc sobre Expectativas Docentes de 60 ítems de opción múltiple. Las preguntas de este instrumento versan sobre las líneas metodológicas más habituales que las personas participantes podrían se- guir a la hora de desempeñar su labor como docentes, así como sobre las características de los centros educativos y el alumnado. La fiabilidad se calculó mediante el alfa de Cronbach, con un resultado de $\alpha=0.81$. Concretamente, en este instrumento encontramos 6 dimensiones: Planteamiento de objetivos, contenidos y recursos didácticos; Expectativas sobre la evaluación educativa; Diseño de actividades; Concepciones sobre aspectos organizativos de un centro educativo; Concepciones sobre la labor (organización) docente; Creencias acerca del alumnado.

\section{Participantes}

En esta investigación han participado un total de 323 personas con titulaciones superiores y estudiantes del Máster en Formación de Profesorado de Secundaria, Bachillerato e Idiomas, siendo el 32\% hombres y el 68\% mujeres. Debido a las características del Máster, las titulaciones de las personas participantes son muy diversas. En la Tabla 1 podemos ver la distribución de las titulaciones según las áreas de conocimiento. Más del 40\% del alumnado posee titulaciones superiores pertenecientes a la rama de Ciencias Sociales y Jurídicas. Las edades de las personas participantes están comprendidas entre los 21 y 58 años, siendo la media 28,5 años. En la muestra estudiada encontramos que un $18,3 \%$ de las personas participantes manifiestan tener experiencia docente previa, mientras que el $81,7 \%$ no ha ejercido nunca docencia en secundaria. Dentro del personal docente con experiencia previa encontramos personas con hasta 12 años de docencia, aunque el 90\% tiene 3 años o menos. En este caso, la media en tiempo de docencia es 1,94 años.

Tabla 1. Titulaciones de los participantes por Ramas.

\begin{tabular}{cc} 
Ramas de conocimientos & Frecuencia \\
\hline Artes y Humanidades & 4,4 \\
Ciencias Sociales y Jurídicas & 41,5 \\
Ciencias & 16,4 \\
Ciencias de la Salud & 15,8 \\
Ingeniería y Arquitectura & 21,9 \\
\hline
\end{tabular}

\section{Resultados}

A continuación se recogen los resultados organizados según los objetivos específicos propuestos en la investigación desarrollada:

Objetivo 1: Analizar los distintos elementos didácticos que plantea el profesorado novel: objetivos, actividades, contenidos y recursos didácticos.

Para tratar de dar respuesta a este primer objetivo, a continuación se presentan los resultados correspondientes a la primera dimensión del instrumento: planteamiento de objetivos, contenidos y recursos didácticos. También responde a este objetivo la dimensión del cuestionario relativa al diseño de actividades.

\section{Objetivos y contenidos}

Según los resultados obtenidos, a la hora de establecer los objetivos del proceso de enseñanza, el profesorado novel valora positivamente el hecho de que el alumnado conozca permanentemente los objetivos, de manera que casi la totalidad de la muestra se divide entre la opción mucho $(47,5 \%)$ y bastante $(47,9)$ para dar respuesta a este ítem.

Encontramos que la mayoría de los participantes prefiere potenciar en el alumnado un aprendizaje significativo por des- 
cubrimiento guiado $(72,2 \%)$ seguido del aprendizaje cooperativo (53,3\%). Por su parte, encontramos que sólo un 5,8\% piensa basar el proceso de enseñanza en un aprendizaje de tipo memorístico por recepción.

Por otro lado, la modalidad de trabajo para llevar a cabo más a menudo con el alumnado es aquella que implica trabajar en $p e-$ queño grupo (41,7\%), grupo mediano $(41,7 \%)$ y gran grupo $(29,7 \%)$, seguidos de la modalidad individual (26,6\%).

La organización del grupo-clase que con más frecuencia creen que utilizarán será aquella que implique situar al alumnado dentro del aula en forma de " $U$ " $(57,1 \%)$, seguida de las líneas o filas (32,4\%), de los pequeños grupos (32\%), de la estructura circular $(17,4 \%)$ y finalmente sólo un $6,9 \%$ prefiere que el estudiante se posicione de forma individual dentro del aula.

Al preguntar a los participantes por cuáles son sus preferencias para estructurar el trabajo a lo largo de una hora de clase, encontramos que las opciones predominantes son las siguientes (ver Figura 1):

Figura 1. Estructura de trabajo en el aula.

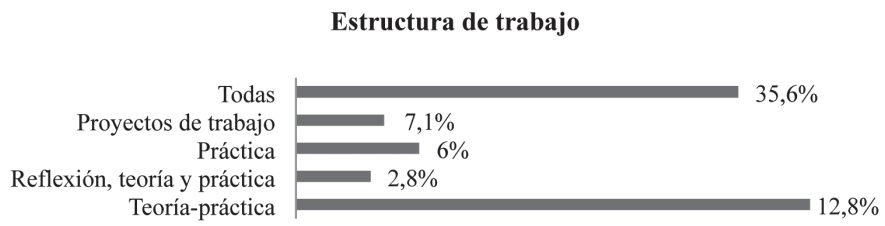

Como se observa en la Figura 1, un número importante apuesta por la combinación de distintas estructuras para abordar el trabajo en el aula $(35,6 \%)$ siendo ésta: explicación y ejercicios, actividades y corrección, proyectos de trabajo, investigación y puesta en común. Sin embargo, el 12,8\% muestra preferencias por la estructura Teórico-práctica en la que el docente explica en primer lugar los contenidos teóricos y posteriormente plantea una serie de ejercicios o actividades. Un 6\% de los participantes manifiesta que abordará los contenidos únicamente a través de actividades y un $7,1 \%$ a través de Proyectos de trabajo. Finalmente, nos encontramos con un porcentaje menor (un 2,8\%) que representa a quienes consideran preciso promover la reflexión y curiosidad del alumnado a través de cuestiones o actividades iniciales que se realizarán de manera previa a la exposición de los contenidos teóricos y de las consecuentes actividades prácticas.

Independientemente de la estructura de trabajo, la mayoría de los futuros profesionales (98\%), señalan que insistirán, mediante ejemplos concretos, para qué puede ser útil lo que se va a enseñar, y que permitirá que el alumnado intervenga cuando lo desee (83,3\%) frente a una minoría que sólo permitirá la intervención del alumnado a su requerimiento $(13,9 \%)$.

Por su parte, un 72,8\% del profesorado novel entiende que el hecho de hacer bromas o referencias personales durante el desarrollo de la clase, promoverá un ambiente afectivo y relajado entre el alumnado, a diferencia del $26 \%$ que estima que este hecho ocasionará revuelo y falta de respeto.

En lo referente a la forma de presentar los contenidos, tanto teóricos como prácticos, obtenemos un predominio entre el profesorado la modalidad audiovisual (73\%), seguida de las recogidas en la Figura 2:
Figura 2. Presentación de contenidos. Distintas modalidades.

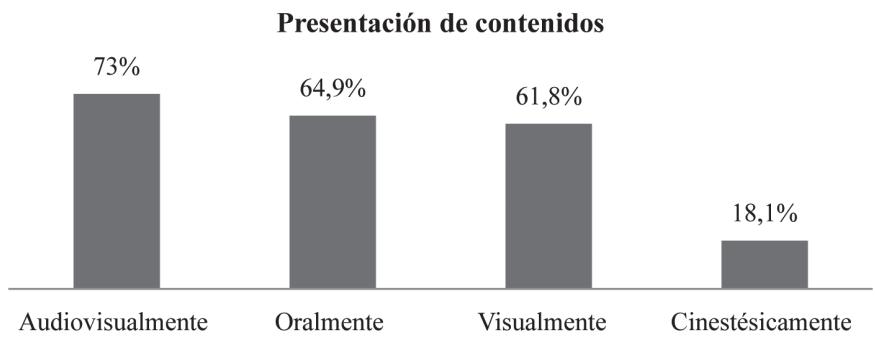

\section{Recursos didácticos}

Con respecto a los recursos didácticos a utilizar, los participantes piensan que para enseñar, generalmente, utilizarán en primer lugar sus propios recursos de elaboración propia $(76 \%)$, seguido de los que les proporcione el Departamento Didáctico en cuestión $(60,9 \%)$. Entre los materiales que utilizarán con mayor frecuencia destacan los libros de texto $(74,9 \%)$, los equipos informáticos $(68 \%)$, los vídeos $(45,2 \%)$ frente a otros como los libros de lectura $(28,6 \%)$ y la televisión (14\%). Además de lo anterior, hallamos que gran parte de la muestra considera que utilizará los libros de textos como materiales de apoyo (71,8\%) frente al 27,8\% que estima que los usará en ciertas ocasiones como material de consulta. Por último, un $21,8 \%$ cree que los utilizará como recursos necesarios para desarrollar el programa de la asignatura.

\section{Actividades}

En relación a los resultados sobre el diseño de actividades cabe destacar que la actividad desarrollada con mayor frecuencia será de introducción-motivación con un $84,5 \%$ seguida por evaluación con un $63 \%$ mientras que las actividades menos frecuentes serán las de ampliación $21 \%$. El resto de actividades como la detección de conocimientos previos, desarrollo, síntesis-resumen, consolidación, recuperación, y refuerzo, serán llevadas a cabo tanto a veces como casi siempre en una proporción similar (ver Figura 3).

Figura 3. Diseño de actividades.

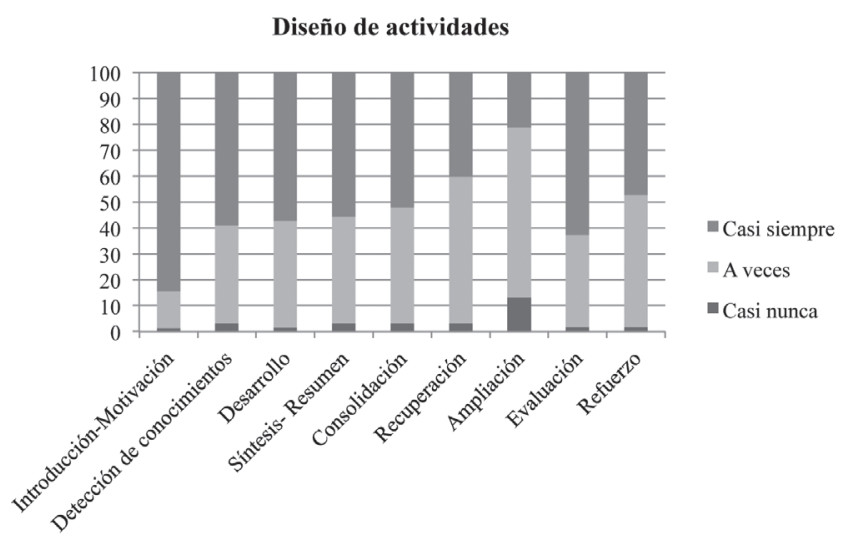

Respecto a la duración que tendrán las actividades que el profesorado plantee en su labor cotidiana, los tiempos cortos y dinámicos y las actividades de entre 15 y 30 minutos acumulan un porcentaje de $86,6 \%$ frente al $13,4 \%$ que piensa que planteará actividades con una duración aproximada de un periodo lectivo o incluso más. El nivel exigido de atención y concentración al alumnado en clase está comprendido entre nivel medio y alto. Solo un $7 \%$ cree que exigirá un nivel muy alto. 
Por su parte, un 95,3\% del profesorado afirma que organizará actividades fuera del centro educativo para desarrollar algún tema. Respecto al comienzo de las clases con algún tipo de motivación, la mitad del profesorado novel piensa que lo llevará a cabo en ocasiones, mientras que un $36,5 \%$ lo hará siempre.

Un dato muy significativo e importante es que una amplia mayoría $(89,6 \%)$, afirma que normalmente tendrá diseñadas distintas actividades graduadas en dificultad. Del mismo modo, un 94\% planteará a su alumnado trabajos de investigación que serán desarrollados tanto dentro como fuera del aula. Estos trabajos serán de carácter individual en un $47 \%$, mientras que un $27 \%$ serán grupales y un $25 \%$ combinarán tareas individuales y grupales. Además, tendrán un carácter tanto de ampliación como de base para el estudio.

Objetivo 2: Caracterizar las expectativas del profesorado novel sobre evaluación educativa

En cuanto al objetivo 2 de la presente investigación, encontramos diferentes resultados que nos indican las expectativas que posee el grupo de participantes sobre la evaluación educativa. En primer lugar, podemos señalar que aproximadamente la mitad de ellos (50,2\%) establecerá la evaluación como recurso periódico para comprobar el avance del alumnado y el 43,1\% evaluará de manera periódica aunque también realizará una comprobación al final de cada trimestre. Sólo un 6,7\% afirma que realizará únicamente una comprobación final.

Al preguntar al profesorado novel si verificará periódicamente si cada uno de los alumnos y alumnas realizan aprendizajes significativos y comprensivos, hallamos que el 76,5\% lo hará siempre, el $21,5 \%$ sólo a veces y un $2 \%$ estima que no lo verificará. Las principales formas mediantes las que se verificarán los aprendizajes son las siguientes: un 17,2\% utilizará los exámenes, un $16 \%$ planteará cuestiones al final de cada unidad didáctica y el $14,4 \%$ de la muestra propondrá al alumnado distintas actividades (grupales e individuales) para comprobar los aprendizajes significativos. Por último, un 2,8\% hará dicha comprobación a través de distintas exposiciones orales que el alumnado deberá realizar sobre lo aprendido.

En los casos en los que el profesorado deba corregir a sus estudiantes, encontramos que un 58,9\% apuesta por realizar las correcciones de manera grupal frente a un 51,3\% que pretende hacerlo individualmente. Un 9,8\% corregirá tanto grupalmente como de manera individual. Además, al realizar dichas correcciones un $16,7 \%$ cree que señalará únicamente lo positivo de las respuestas mientras que un $81,3 \%$ indicará tanto lo aspectos positivos como los errores. Solo un $2 \%$ se parará exclusivamente en los errores.

Por otro lado, al plantear a los participantes qué conductas (académicas y sociales) recompensarán prioritariamente, obtenemos que el trabajo personal es la conducta que más recompensarán $(85,7 \%)$ seguidas de las siguientes (ver Tabla 2$)$ :

Tabla 2. Conductas académicas y sociales a recompensar.

El trabajo personal: $85,7 \%$

El trabajo en equipo y la colaboración en el mismo: 74,9\%

El respeto a los demás compañeros y al trabajo de los mismos: $71,4 \%$

El respeto al profesorado y a la institución: $57,1 \%$

El rendimiento: $38,2 \%$

La atención durante las explicaciones: $34 \%$

La realización de tareas y ejercicios para casa: $25,5 \%$

El cuidado de los materiales y la presentación de trabajos: $20,8 \%$

Un número alto de los participantes (el 81,1\%) recompensará de manera inmediata las conductas positivas anteriores, mientras que el 18,4\% lo hará una vez que haya transcurrido un tiempo. La forma de recompensar o reforzar dichas conductas son varias, entre las que destacan elogiar al alumnado públicamente $(68,1 \%)$ y realizar comunicaciones positivas a los familiares de éste (51,4\%). En la Tabla 3 podemos ver otros modos de recompensar las conductas positivas:

Tabla 3. Modalidades de recompensa o refuerzo positivo.

Mediante elogio público: $68,1 \%$

Mediante comunicaciones positivas a la familia: $51,4 \%$

Mediante calificaciones o anotaciones en sus trabajos o cuadernos: $49,4 \%$ Mediante elogio privado: $42,1 \%$

Mediante refuerzos de actividad: $28,6 \%$

Mediante refuerzos sociales: $13,9 \%$

Mediante refuerzos materiales: $5,8 \%$

En otro sentido, hallamos que las conductas que el profesorado novel estima que recriminará o castigará son las que aparecen a continuación (ver Tabla 4):

Tabla 4. Conductas académicas y sociales a recriminar o castigar.

No respetar a los demás compañeros: 79,9\%

La falta de respecto al profesorado y a la institución: 76,1\%

La escasez de trabajo personal: $67,8 \%$

No participar o bloquear los trabajos en equipo, interrumpiendo a los demás: $57,5 \%$

No cuidar los materiales propios, de los compañeros o del centro: $33,6 \%$ La escasez de rendimiento: $27 \%$

Bajo nivel atención durante las explicaciones o distracción: 26,3\% La no realización de tareas extraescolares y ejercicios para casa: $16,2 \%$ La presentación inadecuada de sus trabajos: 13,9\%

Dichas conductas se recriminarán de forma inmediata según el $87,8 \%$ de la muestra y después de que haya transcurrido un tiempo según el 12,2\% restante. El modo principal en que el profesorado prevé que llevará a cabo las recriminaciones será a través de una llamada de atención al alumnado de manera privada, aunque también encontramos otros modos de recriminación (ver Tabla 5).

Tabla 5. Distintas modalidades de recriminación o castigo.

Mediante recriminación en privado: 74,1\%

Mediante comunicaciones a su familia: $49,4 \%$

Mediante calificaciones o anotaciones en sus trabajos o cuadernos: $25,1 \%$ Mediante recriminación pública: $21,2 \%$

Mediante castigo de actividad: $15,8 \%$

Mediante la utilización de la ironía como medio de estimulación: 14,3\% Mediante trabajos extras: $14,3 \%$

Mediante expulsiones o mandándole a su tutor u orientador: $11,6 \%$

Mediante castigos sociales: $6,6 \%$

En lo referente a la contingencia y cumplimiento de los castigos, hallamos que el 43,6\% siempre los hará cumplir, el 31,1\% sólo a veces y el $12 \%$ dice que no lo hará nunca.

Objetivo 3: Identificar las ventajas e inconvenientes de la profesión docente según el profesorado novel

Dando respuesta al último objetivo de esta investigación, descubrimos que las principales ventajas que los participantes piensan que vivirán como docentes son: la propia satisfacción personal al poder llevar a cabo su vocación profesional (un $16 \%$ indica esta opción), y la gratificación tras comprobar la evolución y aprendizaje del alumnado (con un 12,8\%). Otras ventajas señala- 
das son: la estabilidad económica (6,4\%), el horario laboral (4,4\%), el periodo vacacional $(4,4 \%)$ y el apoyo del resto del profesorado $(2,8 \%)$.

Por otro lado, tal y como podemos observar a continuación en la Tabla 6, los inconvenientes prioritarios señalados son la falta de respeto del alumnado hacia el docente y la desmotivación de los estudiantes.

Tabla 6. Inconvenientes según el profesorado novel.

Falta de respeto por parte del alumnado: $15,6 \%$

Desmotivación del alumnado: 7,2\%

Diversidad del alumnado: $4,4 \%$

Estrés (ante la gestión de conflictos): 4\%

Agresiones verbales o físicas hacia el docente: $3,2 \%$

Falta de compañerismo por parte del profesorado: $3,2 \%$

Alumnos disruptivos: 2,8\%

Falta de respeto por parte de las familias del alumnado: 2,8\%

Por último, teniendo en cuenta todos los resultados anteriores podemos caracterizar las concepciones metodológicas del profesorado novel (objetivo general de la investigación) como innovadoras, actuales y adecuadas a las realidades escolares. Ello lo avala el hecho de que la mayoría de los participantes abogue por un aprendizaje significativo, basado en el establecimiento de objetivos consensuados con el alumnado, con una metodología participativa ayudada por una distribución espacial en $U$ que propicia la interacción y participación, dado el contacto permanente y total de todos los miembros de la clase. Asimismo el paradigma constructivista destaca en las concepciones cuando, se afirma que las dificultades en las actividades, elemento didáctico fundamental, se diseñarán de forma gradual de forma que tengan cabida todas las situaciones en las que el alumnado pueda encontrarse.

\section{Discusión y conclusiones}

A partir de los resultados, la mayoría de los participantes manifiestan una posición docente abierta a la investigación, al aprendizaje constructivo y al descubrimiento por parte del alumnado, como ya recogían Genovard y Gotzens (1990) con esta tendencia progresista encontrada en su investigación.

De igual manera, participamos con Feixas (2004) en que no tiene porqué la experiencia docente conllevar un mayor posicionamiento docente que se encamine a conseguir aprendizaje significativo, de hecho nuestros participantes adolecen de experiencia y sin embargo ese es su planteamiento docente.

Por su parte, autores como Clark y Peterson (1990) consideraban que la conducta de los docentes era la consecuencia de sus pensamientos, expectativas, etc., y nosotros encontramos una amplia mayoría de profesorado que cree positivo abrirse personalmente al alumnado, mediante bromas o comentarios personales porque le propiciarán un ambiente relajado y más cordial así como permitirán que el alumnado participe cuando lo desee. Siguiendo en esta línea, también encontramos que una amplia mayoría del profesorado participante pretende trabajar con materiales elaborados por ellos mismos, una muestra más de la disposición a implicarse y volcar sus posicionamientos y pensamientos personales.

Una de las conclusiones más llamativas y alentadoras de nuestro estudio es el uso que el profesorado participante declara que dará a la evaluación, siendo como es, un elemento didáctico tan importante y potente, a la vez que tradicionalmente configurado. Gran parte declara que usará la evaluación de forma permanente, continua, para verificar los progresos del alumnado, siendo una minoría quien sólo la utilizará en momentos finales del proceso de enseñanza, como comprobación de aprendizajes terminados.

Asimismo, Sánchez y Boix (2008) afirmaban que las mayores preocupaciones de los docentes noveles eran la adecuación al alumnado y ser capaz de innovar. En nuestra investigación, el profesorado participante muestra mayoritariamente una organización de clase que comience con actividades motivadoras que "enganchen", un seguimiento continuo de los avances para no propiciar desmotivación y el diseño de actividades externas al aula, así como variados materiales y recursos que dan muestras de innovación y apertura docente.

Es muy interesante destacar también una conclusión referida al sistema de refuerzos al alumnado. La gran parte del profesorado novel cree que reforzará inmediatamente los logros, siendo el esfuerzo personal lo que más valora. Asimismo, recriminará conductas negativas de forma inmediata, fundamentalmente la falta de respeto al alumnado o profesorado, siendo la modalidad de realización de forma individual y contando con la familia.

Concluimos, en consonancia con lo expuesto por Beca y Boe$\underline{\operatorname{rr}(2008)}$ que el profesorado de nuestra investigación constituye un magnífico potencial renovador para el sistema educativo y su usuario más importante, el alumnado; la incorporación de docentes jóvenes, ilusionados y motivados puede hacer realidad los cambios educativos tan traídos y llevados por las distintas leyes educativas, como también aportan Azcárate y Cuesta (2005). También otros investigadores como Huberman (1996) apuntan en la misma línea al defender la tesis de una necesaria actitud colaboradora para la mejora de los centros, concordando con nuestros resultados que arrojan una mayoría de participantes dispuestos a usar los materiales del departamento didáctico al que pertenezcan, dando muestras de este aperturismo grupal.

Otra de las conclusiones que se señalan, se ajusta a los hallazgos de Veenman $(1984,1988)$ para quien dos de los inconvenientes fundamentales de la profesión docente, desde la óptica del profesorado novel, son la disciplina y la motivación, siendo asimismo una preocupación encontrada por Whitworth et al. (1996) respecto al control del alumnado.

Al margen de los hallazgos y consideraciones expuestas en líneas previas, se finaliza este artículo haciendo constar alguna de las limitaciones y propuestas de mejora de la presente investigación. Así, convendría procurar convertirla en un estudio secuencial mediante el que continuar el proceso de conocimiento acerca de estos profesionales y comprobar, de esta manera, el grado de acuerdo o desacuerdo apreciado al comparar sus expectativas docentes en los momentos iniciales de su profesión y las realizaciones concretas una vez situados en la realidad educativa de las aulas. Asimismo entendemos que se debería ampliar la muestra a otros contextos que permitieran generalizar estos resultados.

Confiamos pues que, en la misma línea argumental que investigadores como Al-Musawi (2001) y Cámara (2010), que los profesionales que se enfrentan a una situación tan novedosa e importante en sus vidas como es el comienzo de una vida laboral, enseñanza con adolescentes y jóvenes, mantengan vivas durante mucho tiempo sus ilusiones, creencias, expectativas y pensamientos que en estos momentos, de forma taxativa se encuentran en modelos actuales, constructivistas, innovadores, cooperativos y centrados en el alumnado, como protagonista de la educación.

\section{Referencias bibliográficas}

Al-Musawi, N.M. (2001). Psychometric properties of the Beck Depression Inventory-II with university students in Bahrain. Journal of Personal Assessment, 77, 568-579. 
Azcárate, P., y Cuesta, J. (2012). Factores que facilitan el cambio en el profesorado novel de Secundaria. Revista de Educación, $357,327-350$.

Beca, C.E., y Boerr, I. (2008). El proceso de inserción a la docencia. En AA.VV: Aprendizaje desarrollo profesional docente (pp. 109-118). Madrid: Santillana.

Capell, K. (1998). Inclusive preschool programs: Teachers' beliefs and practices. Early Childhood Research Quarterly, 13(1), 87- 105.

Casado, A. (2007). Creencias de los estudiantes de magisterio sobre las interacciones en el aula. Ávila: Ediciones de la UCLM.

Cámara, A. (2010). Valores en futuros profesores de Secundaria. REIFOP , 13(3), 113 - 121.

Clara, A. (2016). ¿Enseño como aprendí?: el rol del estilo de aprendizaje en la enseñanza del profesorado universitario. Aula Abierta, 44(2) 91-98.

Clark, C. M., y Peterson, P.L. (1990). Procesos de pensamiento de los docentes. En M. C. Wittrock, La investigación de la enseñanza. Profesores y alumnos (pp. 443- 539), vol. III, $3^{3}$ ed. Barcelona: Paidós Educador.

Doménech, F., y Gómez, A. (2003). Las creencias psicopedagógicas de los futuros profesores de secundaria y su relación con las demandas de examen y con la organización espacial de la clase. Revista de Investigación Educativa, 21(2), 489-505.

Feixas, M. (2004). La influencia de factores personales, institucionales y contextuales en la trayectoria y el desarrollo docente de los profesores universitarios. Educar, 33, 31-59.

Fuller y Brown, O. (1975). Becoming a teacher. En Ryan (Ed). Teacher Education (25-52). Chicago: NSSE.

García, J. Orellana, M.C., y López-Cepero, J. (2010). La experiencia personal como elemento de reflexión sobre la práctica docente. Revista de Enseñanza universitaria, 35, 4-15.

Genovard, C. y Gotzens, C. (1990). Psicología de la Instrucción. Madrid: Santillana.

González, M. (2012). Pensamiento del profesor novel de inglés. Acercamiento y análisis de su problemática durante el periodo de iniciación profesional: un estudio de caso autobiográfico. Tesis doctoral. Facultad de Educación y Trabajo Social. Valladolid.

González-Granda, J. F., Álvarez-Menéndez, M. L., y González-Pienda, J. A. (1991). El profesor principiante. Análisis de sus problemas. Magister, 9, 119-128.

Huberman, M. (1996). Focus on Research: Moving Mainstream: Taking a Closer Look at Teacher Research. Language Arts, 73(2), 124-140.

Kelly, G. A. (1969). Clinical psychology and personality: The selected papers of George Kelly. Oxford: John Wiley y Sons.

Marcelo, C. (1993). El primer año de enseñanza. Análisis del proceso de socialización de profesores principiantes. Revista de Educación, 300, 225-277.
Marcelo, C. (1987). El pensamiento del profesor. Barcelona: CEAC.

Marcelo, C. (2008). Evaluación de la calidad para programas completos de formación docente a través de estrategias de aprendizaje abierto ya distancia. RED. Número monográfico VII.

Mayor-Ruiz, C. (1996). Los problemas y necesidades de los profesores principiantes universitarios. Bordón, 1(48), 27-71.

Melief, K. Tigchelaar, A. y Korthagen, F. (2010). Aprender de la práctica. En O. Esteve, K. Melief y A. Alsina, Creando mi profesión. Una propuesta para el desarrollo profesional del profesorado (pp.19-37). Barcelona: Octaedro.

Negrillo, C., y Iranzo, P. (2009). Formación para la inserción profesional del profesorado novel de Educación Infantil Educación Primaria y Educación Secundaria: hacia la reflexión desde la inducción y el soporte emocional. Profesorado. Revista de Currículum y Formación del profesorado, 13(1), 157-182.

Pérez-Gómez, A.I. (1987). El pensamiento práctico del profesor. Vínculo entre la teoría y la práctica. Revista de Educación, 284, 199-222.

Sánchez, A., y Boix, J. L. (2008). Los futuros profesores de Educación Secundaria: inicio de su profesionalización y construcción de su identidad docente. REIFOP, 11(2).

Shulman, L.S. (1990). Paradigmas y programas de investigación en el estudio de la enseñanza: una perspectiva contemporánea. En M.C. Wittrock, La investigación de la enseñanza. Profesores y alumnos, vol. III, 3ª ed. (pp. 9-91). Barcelona: Paidós. Educador.

Valdemoros-San Emeterio, M. A., y Lucas-Molina, B. (2014). Competencias que configuran el perfil del docente de primaria. Análisis de la opinión del alumnado de Grado en Educación Primaria. Aula Abierta, 42, 53-60.

Veenmam, S. (1984). Perceived problems of beginning teachers. Review of Educational Research, 54, 143-178.

Veenman, S. (1988). El proceso de llegar a ser profesor: un análisis de la formación inicial, en Villa, A. (coord.). Perspectivas y problemas de la función docente, (pp. 39-68). Madrid: Narcea.

Whitworth, A. B., Oberbauer, H., Fleischhacker, W. W., Lesch, O. M., Walter, H., Nimmerrichter, A., y Potgieter, A. (1996). Comparison of acamprosate and placebo in long-term treatment of alcohol dependence. The Lancet, 347(9013), 1438-1442.

Zeichner, K.M. (1983). Alternative Paradigm of Teacher Education. Journal of Teacher Education, 34(3), 3-9.

Zeichner, K.M. (1993). A formação reflexiva de professores: ideias e práticas. Lisboa: Educa. 